\title{
La vinculación en nuestros días: entre el peso de lo institucional y el paso de la pandemia
}

\author{
The linkage nowadays: Among the institutional relevance and the crossing through the \\ pandemic
}

Mehdi Mesmoudi

Universidad Autónoma de Baja California Sur m.mesmoudi@uabcs.mx

La Paz, B.C.S., México

Cómo referenciar:

Mesmoudi, M. (2022). La vinculación en nuestros días: entre el peso de lo institucional y el paso de la pandemia. Emerging Trends in Education, (4)8A, 94-106

https://doi.org/10.19136/etie.a4n8A.4716

Disponible en:

https://revistas.ujat.mx/index.php/emerging

DOI:

https://doi.org/10.19136/etie.a4n8A.4716

Recibido: Aceptado:

Publicado:

$13 / 09 / 2021$

09/12/2021

05/01/2022

\section{Resumen:}

A raíz de la pandemia, la Universidad se ha vuelto un espacio predilecto para las innovaciones sociales e institucionales donde el talento humano y la transformación digital son el insumo ideal para la revolución de nuestro tercer milenio. Este escenario de efervescencia se enmarca en las vislumbres que la Responsabilidad Social ha establecido como filosofía institucional transversal en las últimas dos décadas y que, entre otras cosas, exige un mayor protagonismo social de la Universidad en dichos cambios sociales, políticos y económicos.

Este texto trata de formular los distintos mecanismos interinstitucionales para impulsar un modelo de vinculación orientado a la RSU cuya dimensión descansa en la internacionalización, los valores interculturales, la diplomacia cultural y la innovación digital que dinamiza los vínculos que la Universidad puede establecer y fortalecer su proyección transoceánica como su papel activo en la nueva cartografía de la cooperación internacional.

Palabras clave: Responsabilidad Social Universitaria; participación social; interculturalidad; diplomacia cultural; vinculación digital.

\begin{abstract}
:
As a result of the pandemic, the University has become into a preferred space for either social and institutional innovation. In this endeavors, we see human talent and digital transformation being the ideal supply to revolutionize our third millennium, which; is an essential element for the current social, politic and economic turn of.

This unsettled and volatile scenario, put in a frame the inkling that Social Responsibilities settled for cross-wise institutional philosophy. Such discerns, for the last two decades, entail a major social limelight from the part of the University concerning those changes.

The main purpose of this text, is to expound the range of interinstitutional mechanisms to promote a model of binding, oriented to the aspect of internationalization, cultural values, diplomacy and digital innovation of the University Social Responsibility (USR). It is the emphasis of this text, to invigorate the ties that the University can establish and strengthen on its transoceanic dimension, as well as its active rol in the new international cooperation cartography
\end{abstract}

Keywords: University Social Responsibility (USR); social involvement; interculturality; cultural diplomacy; digital connection. 


\section{La vinculación en nuestros días: entre el peso de lo ins- titucional y el paso de la pandemia}

\section{| Introducción}

La pandemia actual inaugura el tercer milenio. Nadie hubiera vislumbrado una hecatombe semejante. La actualidad invita a repensar su incorporación al día a día, como parte del corpus doctrinal de usos, hábitos, costumbres y actitudes que adornan la costra social tanto individual, grupal como societal.

En estos casi dos años, la Universidad ha pasado repentinamente de del acartonamiento institucional a incorporar urgente y tácitamente toda una serie de estrategias, medidas y tips para sobrevivir ante los primeros embates de la pandemia y los últimos suspiros de una institución que quedó anclada en el siglo pasado. Ha incorporado herramientas adquiridas en un tiempo que ni reconoce, como una suerte de "caja negra" de hábitos, sentido común y resonancias magnéticas de un mundo en ruinas aunque, todavía, poblado de "espíritus en alerta y mentes vanguardistas" que todavía creen en el cambio y que dichos cambios pueden y deben marcar la diferencia; es decir, una memoria que ya no mira hacia atrás como un gesto escatológico de la historia, sino de una observación que apunta hacia el presente, contemplando lo que merece ser reconocido, identificado e incorporado como insumo vital, sociocultural y político de la tradición que debe ser revisitada, discutida y construida cotidianamente, en el ejercicio de una democracia participativa, con equidad, con justicia social, con el principio de un pensamiento sostenible, con el espíritu de una profunda co-responsabilidad.

La Universidad, durante esta pandemia, ha transitado por un duro proceso de autorreflexión, animada en gran medida-insisto-por los impulsos vitales de sobrevivencia y, por otro lado, bajo el síntoma filosófico-institucional de la Responsabilidad Social Universitaria que exige una profunda transformación de esta institución en un organismo vivo, dinámico, crítico, innovador y, antes que nada, útil y competente. En pocas palabras, una Universidad que plantee escenarios para enfrentar los desafíos más acuciantes de nuestra humanidad en todos sus dominios.

\section{Apuntes sobre la Responsabilidad Social Universitaria}

Aunque la Universidad se remonta a más de un siglo, es hasta en las últimas décadas cuando se puede observar una genuina y profunda transformación en su razón de ser: su condición social como organismo capaz no solo de especular en torno a los posibles cambios que puede sobrevenir a la humanidad, sino aplicar dichos instrumentos y herramientas del conocimiento en la resolución de los desafíos actuales. La universidad al igual que la lengua si recordamos las palabras del gramático castellano Elio Antonio de Nebrija (1492) también ha sido "compañera del imperio" en un tiempo y espacio determinados. La Universidad históricamente ha sido objeto de un sinfín de modificaciones hasta convertirse hoy en lo que es: una entidad poderosa hecha de tiempo que desafía el tiempo mismo.

Conviene, sin embargo, deconstruir el legado, dejando atrás la visión eurocéntrica que sigue sosteniendo que la Universidad de Bolonia es la primera institución de educación superior en ser fundada cuando, por el contrario, al menos existen tres universidades orientales -Al Qarawiyyin (859) en Marruecos, Al-Azhar (975) en Egipto y la Nizamiya a principios del siglo XI en Irán- que al igual que en el sistema cristiano del Studium Generale 
cumplían en ser centros abiertos al mundo, impartían estudios superiores y contenían las artes (retórica, historia, filosofía, lógica), la teología (Corán, Hadiz, sharia y tafsir), la medicina y el derecho (Chebel, 2011). Más allá de la diferencia religiosa -concebida epocalmente como rivalidad bélica y hostilidad discursiva- el vocablo árabe de "madrasa" designaba cualquier institución -donde se enseñaba especialmente las cuestiones enfocadas al Corán y la tradición profética-no debería ser un obstáculo epistémico ni suponer por ello un sesgo en la mirada sociocultural del fenómeno.

La Responsabilidad Social Universitaria (RSU) cuya base descansa en la ecología de saberes y un pensamiento sostenible cuestiona los criterios de la Organización de las Naciones Unidas para la Educación, la Ciencia y la Cultura (UNESCO) que considera la institución de la "madrasa" como una universidad incipiente o a medias $\mathrm{y}$, en este sentido, habría que proceder desde una dimensión poscolonial y no orientalista, intercultural y no eurocéntrica de la historia porque el conocimiento adquirido desde el Renacimiento hasta la mitad del siglo pasado es fruto del filtro occidentalista del mundo, por ende, hegemónico y excluyente. Con justa razón, la RSU combate el orientalismo institucional que romantiza las comunidades y exotiza su disposición social, cultural y política que devela un multiculturalismo que las reconoce, aunque como ornato paisajístico, carentes de voz, anuladas en su formulación soberana de sus haberes, saberes y haceres.

Las universidades evolucionan históricamente. Con la globalización y la hibridez de las fronteras jurídiconormativas de los estados-nación, estos cambios sociopolíticos deben ser abordados, replanteando las políticas y las estrategias que van más allá de la jurisdicción de un país o de una geopolítica. Estos cambios institucionales, infraestructurales y culturales dan cuenta de una revolución mundial:
Una nueva sociedad surge y cuando pueda observarse una transformación estructural en las relaciones de producción, en las relaciones de poder y en las relaciones de experiencia. Estas transformaciones conllevan una modificación igualmente sustancial de las formas sociales del espacio y el tiempo, y la aparición de una nueva cultura (Castells, 2001, p. 410).

Castells asocia esta cultura contemporánea con "la sociedad red", "la economía informacional/global" y "la cultura de la virtualidad real" (Castells, 2001, p.406) que son un semillero de "la revolución de la tecnología de la información; la crisis económica tanto del capitalismo como del estatismo y sus reestructuraciones subsiguientes; y el florecimiento de los movimientos sociales y culturales, como el antiautoritarismo, la defensa de los derechos humanos, el feminismo y el ecologismo" (Castells, 2001, pp.405-406), que refleja "una cultura juvenil global" (Hobsbawm, 2014, p. 329) en el entendido de que la RSU no solo es un compendio de los daños irreversibles al planeta en todos sus dominios, sino al mismo tiempo ofrece distintos mecanismos de defensa, resistencia y ruptura ante una cultura depredadora y obsoleta.

En este contexto de cambios globales se sitúa la educación superior y, particularmente, las universidades que se han sumado a la toma de decisiones para contrarrestarlos, empezando por trazar conjuntamente con las instituciones gubernamentales una agenda de trabajo común: la agenda 2030 de la Organización de las Naciones Unidas (ONU) que apunta a la sostenibilidad como política intrínsecamente global que se despliega epistémica e ideológicamente en todo el quehacer gubernamental, sociopolítico, económico y cultural. La otra agenda, a nivel nacional en el programa federal Nueva Escuela Mexicana (2018-2024), llama a cerrar las brechas de la inequidad e injusticia sociales. Ambas proyecciones vislumbran a la universidad como espacio de discusión en torno a los 
cambios que deseamos observar en nuestra sociedad: "De lo que hoy se empieza a pensar depende lo que mañana se vivirá en las plazuelas" (Ortega y Gasset, 2002, p. 14). La Universidad se convierte en un laboratorio sociopolítico, cultural y económico de la sociedad del porvenir.

La RSU se entiende como "política integral de gestión universitaria, transversal a todos los procesos, funciones, actividades administrativasy académicas dela institución" (Unión de Responsabilidad Social Universitaria de Latinoamérica [URSULA], 2017) en el sentido de que se forma, se investiga, se vincula, se difunde la cultura y se divulga en aras de estos cambios promovidos a diferentes escalas. La universidad -como organización de orden público- no deja de reflexionar "entre lo que hace y lo que debería de hacer" (URSULA, 2017) y prepara a sus estudiantes para formar "ciudadanos activos por un cambio social" porque nuestra universidad pretende ser "una organización responsable por sus impactos en la sociedad y en el medio ambiental" (URSULA, 2017). Con justa razón, Jacques Derrida (2010) encuentra un vínculo intrínseco entre las nuevas humanidades y la universidad del futuro porque en ambas visiones del mundo se cuestionan las formas en que el ser humano se ha relacionado con el mundo y sus semejantes, construyendo, al mismo tiempo, este umbral crítico en torno a los discursos depredadores que aniquilan el planeta y el sentido de la vida.

\section{Un nuevo modelo de vinculación}

\section{a) HACIA LA PARTICIPACIÓN SOCIAL}

Con el fortalecimiento en el sustento conceptual, filosófico y operativo de la RSU, la extensión que ponía un énfasis en la pertinencia social de la universidad en el sentido de llevar a las comunidades los frutos académicos y de la investigación cede su lugar a la vinculación con una dimensión integral y circular de la interacción de la Universidad con su entorno. Esta relación no se limita a los vínculos Universidad/ empresa, sino que amplía sus redes de colaboración con los demás sectores sociales, especialmente la sociedad civil y las comunidades, anteriormente excluidas de la cadena productivo-desarrollista (Malagón Plata, 2006). Mientras la RSU es concebida como discurso y práctica, la vinculación asumiría su función operativa y, a la vez, es una praxis misma de la RSU. No puede existir una RSU sin vinculación ni viceversa. De acuerdo a lo anterior, se plantea la necesidad de fomentar y concientizar sobre la pertinencia de la educación superior enfocada a los impactos que tiene sobre el desarrollo regional, nacional y local de nuestro país, estableciendo nuestra vinculación estratégica con el sector empresarial, productivo y social (Rodolfo Tuirán, 2013), considerando que "la función social de la universidad constituye la esencia misma de la universidad" (Malagón Plata, 2006, p. 88) cuya relación no solo es técnica sino conceptual.

Con el modelo de RSU, la vinculación asume una amplitud institucional como Participación Social puesto que insiste en la raigambre comunitaria que históricamente la Universidad ha mantenido con sus entornos. Desde esta lógica socio-institucional, la Universidad plantea una serie de retos en los que el seguimiento y los impactos de la vinculación a futuro deben de involucrar aspectos en la formación profesional y el fortalecimiento de la docencia, la investigación y el desarrollo tecnológico, los productos, los servicios y las capacidades institucionales, la divulgación de la ciencia y la difusión de la cultura. De acuerdo con la RSU, transitaríamos de la difusión y extensión de la cultura -en cuanto a la superación de la "segunda falacia" (Vallaeys, 2018) -, por un lado, y de la vinculación, por otro lado, hacia la integración en la función operativa de participación social que amplía las miras de la extensión universitaria y guardaría una mayor y profunda cercanía, colaboración e interacción con los distintos entornos tanto a nivel local, estatal, nacional, 
regional e incluso internacional.

La Participación Social, como labor de campo en cuanto a metodología operativa de la RSU, tiene su lugar en la región de su geografía. Sin embargo, no hay que pensar la región geográficamente, sino desde una dimensión de elección de un marco de trabajo; es decir, transitamos de una territorialidad explícitamente geográfica a una "región de co-aprendizaje y de participación social". Esta región de vinculación se establece previamente y por mutuo acuerdo con los actores participantes de los sectores sociales, públicos y productivos (Malagón Plata, 2006, p. 85); por ende, la labor institucional cotidiana con las comunidades atraviesa una serie de regiones de forma transversal, convirtiendo la geografía territorial en un espacio movedizo de interacciones dinámicas. Con justa razón, el compromiso de la Universidad con el desarrollo local, estatal y regional es decisivo, cotidiano y constante.

La atención de los aspectos antes mencionados requiere de una gestión transversal que establezca políticas y estrategias institucionales en aras de fortalecer la vinculación y su inclusión en los sistemas de reconocimiento y el estímulo de la actividad universitaria; auxiliándose de la difusión y puesta en marcha de los manuales de funcionamiento de los nodos de vinculación con los que cuenta la institución, así como la operación de un sistema que permita el registro y el seguimiento de las actividades de vinculación llevadas a cabo por los instancias académicas y otras dependencias universitarias.

En el ámbito de la capacitación, es importante el fomento de un perfil multidisciplinario con una faceta académicoinvestigativa clave en los procesos de capacitación constante y continua en los temas de innovación y transferencia de tecnologías y de conocimiento, cómo los diálogos de saberes contribuye a la construcción de comunidades híbridas de aprendizaje que deja entrever la participación de la Universidad con sus comunidades , la participación social como énfasis en la recuperación y el fortalecimiento del tejido que mantiene la Universidad como ente vivo con la sociedad y sus entornos.

Otra problemática que envuelve no solo el área de vinculación, sino a toda la Red Universitaria, es la necesidad de una profunda "Transformación Digital" que aportaría, en primer lugar, a la Universidad una mayor proyección internacional y una presencia nacional y regional permanente; y en segundo lugar, impregnaría al área de vinculación de un dinamismo institucional vertebrador que inyecte nuevas prácticas interuniversitarias e interinstitucionales, además de la atención de una comunidad académica más amplia y heterogénea. Ambos aspectos conllevarían a un fortalecimiento de la imagen institucional de la Universidad tanto al interior como al exterior. Sin duda, una universidad del siglo XXI debe abrazar dicha "Transformación digital", presente en la Agenda Digital Educativa que promueven la Secretaría de Educación Pública [SEP] en colaboración con la Asociación Nacional de Universidades e Instituciones de Educación Superior (SEP, 2019).

El modelo de vinculación orientado a la RSU establece una hoja de ruta para el despliegue de una serie de sinergias y acuerdos con los distintos sectores de la sociedad, comprendiendo que la universidad, como un ente social, transita hacia una nueva cartografía interinstitucional, de "carácter heterónomo" (Malagón Plata, 2006, p. 86) al incluir en este escenario un enclave formado por la Universidad, el Estado y la Sociedad en un contexto de transnacionalización en aumento.

\section{b) BINOMIO VINCULACIÓN/INTERNACIONALIZACIÓN}

Es innegable que la globalización y la interconexión de los procesos sociales, políticos, culturales y económicos de los estados-nación ha modificado el sentido 
estricto de lo que entendemos por "geopolítica" y exige vislumbrar lo que Immanuel Wallerstein denomina "sistema-mundo" (2003, pp. 137-333) como un síntoma de radicalización que Anthony Giddens (2002) bautiza como “mundialización" que va más allá de la globalización desde un filtro occidental. Precisamente en esta "modernidad radicalizada", Giddens alude, entre los cuatro elementos constitutivos, a una pérdida de la hegemonía occidental (2002, p. 57) que señala la emergencia de la post-verdad, las inteligencias artificiales, una exposición mayor a la virtualidad, la simulación/simultaneidad de escenarios que se concatenan donde los valores sociales, el tejido de las comunidades, el ámbito de la sociabilidad y de las emociones viven una profunda licuefacción acelerada (Bauman, 2013, pp. 23-32) donde el estrés y la angustia alimentan estos "tiempos de depresión" (Augé, 2015 [2013], p. 16). Nunca antes una época había desconocido tanto el significado de su empresa (Paz, 2014 [1994], p. 544) y que incluso se dirige a una odisea sin sentido (Laïdi, 1999, pp. 80-87).

En este mundo diaspórico transita la Universidad intentando mantener su pertinencia social y cultural donde la vinculación es su insumo más valioso en los tiempos actuales. Hablar de vinculación en un contexto de mundialización con las coordenadas que Wallerstein (2003) asume del sistema-mundo atrae consigo en una dupla institucional la internacionalización donde la primera es una de las funciones sustantivas no solo al interior, sino con los distintos sectores sociales, mientras que la segunda se convierte en una especie de alcance que se puede atribuir a un fruto de la misma vinculación. Por un lado, la vinculación es una labor que se busca y se construye; la internacionalización, en cambio, es una cosecha, un logro que se recoge en el mismo momento en que la vinculación se efectúa. En otras palabras, la vinculación y la internacionalización, si se ejercen con inteligencia y talentos institucionales, pueden llegar a ser simultáneas lo que devela un espacio de interacción, interrelación y retroalimentación de tal manera que se necesitan, como un binomio irreductible que exige de una dialéctica intra-institucional transformadora.

La reflexión anterior amerita otra que nos permita esclarecer un elemento conceptual y, por tanto, institucional. Al igual que la RSU, la igualdad de género, los derechos humanos, la interculturalidad, la internacionalización no es un componente que exige la declaración de buenas intenciones y, posteriormente, la presentación de un informe con los indicadores de desempeño atribuibles a dicho componente de internacionalización. La internacionalización es una forma de mirar el mundo, de estar y ser en comunidad, de transformar los ecosistemas y los modos de vida desde un contexto comparado de tal forma nos permita observar cómo es que dichas experiencias son desplegadas en otros entornos por otras organizaciones. La internacionalización deja atrás la perspectiva etnocéntrica y promueve el diálogo con sus comunidades. Nos enfrentamos, por tanto, a una visión integral que toma en consideración la formación educativa, la investigación, la participación social y la gestión y evaluación institucional. Todo lo anterior debe estar atravesado de forma transversal por la interculturalidad, los derechos humanos, la inclusión y la igualdad de género en aras de alcanzar una Universidad sostenible.

La internacionalización es una estrategia multidireccional de gran proyección en nuestros días y en diferentes escalas que hace visible a la Universidad en numerosos foros, organismos y consorcios regionales y globales. Además de la participación social, la internacionalización hace frente al inmovilismo y al aislamiento social y cultural (Mesmoudi, 2021). La internacionalización y la vinculación son dos caras de una misma moneda a tal grado de que la Universidad forma parte del "sistemamundo" al que se refiere Wallerstein (2003). En otras palabras, la internacionalización es un signo de mayoría 
de edad, “intensificación" (Giddens, 2002), plenitud, fortaleza y proyección de la Universidad en el mundo.

\section{c) LA DIPLOMACIA INTERCULTURAL EN LA COOPERACIÓN INTERNACIONAL}

La diplomacia es la labor gubernamental de representar un país en el exterior, estableciendo unas estrategias de acercar las culturas y los pueblos. La diplomacia es el arte de construir una sinergia que toma en cuenta las tradiciones culinarias y musicales, la riqueza arqueológico-histórica, la memoria escénico-visual, el patrimonio literario y mediático, los valores artísticos como usufructo de las relaciones internacionales basadas en el respeto mutuo, el conocimiento del otro y sus especificidades sociales y culturales, el entendimiento y el intercambio inmaterial como formas de dinamizar el desarrollo económico y político propio y de sus socios, basado en una afinidad de elementos dispares complementarios. Aunado a lo anterior, es pertinente recordar que "la cultura es un recurso para la cohesión social, el diálogo entre los pueblos, la paz social y el desarrollo compartido" (Rodríguez Barba, 2015, p. 37).

La diplomacia cultural, no obstante, se distingue de la diplomacia por su desvinculación del área desde donde se despliega dicha labor. Es desde las universidades y las asociaciones civiles donde la diplomacia cultural se configura en aras de construir una serie de vínculos, diálogos e intercambios que están ajenos al discurso gubernamental y se alimentan de un campo semántico y simbólico más amplio y heterogéneo. Octavio Paz había considerado al poeta y editor Carlos Barral "una estación de correspondencias" (2014, p. 977) puesto que había realizado la enorme labor de acercar el público lector europeo a la literatura hispanoamericana; es decir, fungió como puente de transmisión de un patrimonio literario inicialmente latinoamericano que es, hoy en día, herencia universal. Rubén Darío, Alfonso Reyes, José Gaos,
Ortega y Gasset, Carlos Fuentes, Miguel León-Portilla y el propio Octavio Paz son ejemplos (pero hay tantísimos y tantísimas más) de un itinerario que se enmarca en el discurso de la diplomacia cultural que se despliega desde el campo de la literatura y el pensamiento.

Además del ámbito literario y cultural, la Universidad es un campo fértil para impulsar la diplomacia intercultural desde sus distintos nodos de expresión, como los Cuerpos Académicos que se dedican a explorar los pueblos originarios de México y América Latina o estudiar el proceso de la innovación en la región de Asia-Pacífico o bien escudriñar los nexos histórico-antropológicos, sociológicos y culturales de al-Ándalus y la herencia sefaradí con el mundo hispánico; o desde los laboratorios de investigación que rastrean los fósiles de un tiburón o las ruinas de un pueblo mágico; o desde las iniciativas de los estudiantes más jóvenes que están atraídos por la cultura japonesa y surcoreana, entre otros índices. Lo anterior es una muestra evidente de que la Universidad debe abrirse camino hacia el exterior, escuchar a su propia comunidad universitaria, establecer jornadas de participación social para tomar nota de cómo dialogan estos distintos integrantes con realidades alternas, reconfiguran nuestro mundo inmerso en una pandemia sin precedentes y cómo vislumbran el espacio de cooperación interinstitucional en plena virtualización acelerada.

La diplomacia cultural es producto de la mundialización. Futao Huang (2007, p.50) no concibe la internacionalización como la manera en que un estado-nación hace frente a la mundialización ni tampoco es su consecuencia, por lo que ambos conceptos son distintos. Mientras la mundialización es "un proceso dinámico de evolución institucional" vinculada a la educación superior, la internacionalización, en cambio, se refiere al "proceso y las consecuencias de una comunicación mundial instantánea que se realiza con las nuevas tecnologías" (p.51). Jane Knight (2003) plantea que la internacionalización transforma el mundo 
de la enseñanza superior mientras que la mundialización modifica el proceso de la internacionalización. Huang afirma que mientras la internacionalización surge en los debates de los 60, la globalización aparece un poco antes de la caída del muro de Berlín que es concebida como "un modèle unique universellement reconnu, transnational et transculturel" (2007, p. 51) de tal forma la diplomacia cultural se nutre de esta cosecha.

En este contexto de supranacionalidad institucional, la internacionalización como motor de la educación superior ha dejado atrás el discurso decimonónico de las organizaciones políticas, el espíritu nacional o la identidad de un estado-nación y promueve los nuevos valores cifrados en los derechos humanos y el libre tránsito y movilidad, la diversidad cultural y la inclusión, la responsabilidad social y la asociatividad comunitaria, la igualdad de género y la interculturalidad como marcos transoceánicos transversales que desde los Objetivos del Desarrollo Sostenible de la Agenda 2030 de la ONU pretende impulsar en aras de la sostenibilidad del planeta y la justicia social.

La Universidad es un laboratorio donde se ensaya una diplomacia intercultural y paralela que dinamiza estos cambios desde abajo; es decir, con un sentido de la tierra y el involucramiento de las comunidades, los colectivos sociales y culturales, las asociaciones civiles y los actores independientes como alternativa $\mathrm{y}$, al mismo tiempo, como un rediseño de la cartografía interinstitucional de sinergias para el desarrollo social y comunitario. Charles Nattier plantea el éxito de la diplomacia tanto pública como cultural siempre y cuando se contemple la importancia de las redes, la enseñanza de las lenguas, la autonomía y especialización de los acotres, y el apoyo y estímulo al desarrollo (2015, pp. 55-65). El futuro de la diplomacia intercultural descansa en el modo en que la Universidad se involucra con estos sectores sociales, el cuerpo diplomático de las naciones, las instancias culturales con las iniciativas de los "corazones de vanguardia, de almas alertas" (Ortega y Gasset, 2002, p. 5).

d) La virtualidad y la vinculación digital

Existe un abuso del concepto de lo virtual. Pareciera que todo debe terminar en una progresiva virtualización lo que permite iniciar con una reflexión que es de doble rasero. Por un lado, se piensa que lo virtual es una dimensión y una extensión de la realidad; por otro, y como consecuencia de la primera hipótesis, toda virtualidad no es más que un reacomodo de viejas usanzas a un contexto actual. Por tanto, es un asunto de mudanza, una nueva crianza. Sin embargo, es preciso zanjar el debate planteando que la virtualidad ni es la realidad ni su derivado, sino algo totalmente distinto. Es conveniente recordar que también lo que llamamos "realidad" es una construcción simbólica del mundo atravesada por una serie de elementos sociolingüísticos, retóricos, culturales, políticos y mediáticos que da cuenta de la imposibilidad de asir lo real y alcanzar a desentrañar su materialidad discursiva. Desde esta dimensión crítica del constructivismo, hay que admitir que tanto la realidad como la virtualidad son dos instancias que se formulan y se reconfiguran constantemente que exigen sus propias herramientas de observación, conservación, transformación y retroalimentación; es decir, es difícil la aplicación de elementos pertenecientes a entornos reales en la virtualidad acelerada de nuestros tiempos.

Planteado lo anterior, la virtualidad se convierte en un nuevo entorno de emergencia que configura la trama integral del mundo actual. Se observa un viraje de positividad de los entornos reales de construcción simbólica del mundo hacia la dimensión virtual con su propia infraestructura tecnológico-mental, sociocultural, filosófico-política y económica. Mientras "el mundo real" se colapsa en su imposibilidad de prosperar hacia su principio de idealidad, la virtualidad se abre camino hacia 
la construcción de otras formas de vida, otros mundos para observar, proyectar y darle sentido. Nos enfrentamos a una profunda y radical disolución del tejido de la vida como la entendíamos anteriormente, y nos adentramos en una civilización plenamente tecnologizada, virtual, que se distribuye ocularmente, resemantiza sus vínculos a través de las pantallas y los portales digitales, hacia el reino indomable de los emoticonos, íconos de la iconoclastia.

Aunque Marshal McLuhan había vislumbrado este advenimiento cultural y político, es (quizá) el filósofo tunecino de origen judío Pierre Lévy el primero en haber observado la emergencia de las nuevas condiciones materiales y laborales vinculadas a las nuevas tecnologías de la información y la comunicación como el teletrabajo, el influjo de la realidad virtual (2007) y cómo es que los datos disponibles iban a estar distribuidos y circulando en una meta-estructura abismal, interconectada por un sistema de hipervínculos. En el mismo año en que Wallerstein (2003) postulaba su "sistema-mundo", Lévy auguraba una inteligencia colectiva que gobernaba nuestro mundo, hiper-tecnologizada con una memoria borgiana, sin límites, que desafía el tiempo y el espacio (Hermoso Villaverde, 2021).

En el marco de la Nueva Escuela Mexicana, la Secretaría de Educación Pública (2019, p. 6) promueve la Agenda Digital Educativa como "un nuevo paradigma de la digitalización de la educación" donde la neuroeducación, la automatización cognitiva de la inteligencia artificial, la robótica en la educación digital, las multiplataformas de contenidos y recursos digitales, la educación a distancia hacen sonar las trompetas de una era de las nuevas competencias digitales que promueve la alfabetización digital que paulatinamente construye una sociedad digital del conocimiento y vislumbra una sociedad en red. Lo anterior no es posible sin el soporte de una BigData como un campo privilegiado que se dedica a la generación, la captura, el procesamiento, el análisis y la visualización de los datos en una red informacional ilimitada (Hernández-Leal et al., 2017).

Por otro lado, la pandemia del covid-19 ha llevado a la humanidad al confinamiento social, provocando una serie de cambios en la educación superior como la intensificación de la tecnología y el uso de plataformas virtuales que ha servido de "aporte para la población que ha requerido apoyo por el aislamiento social" (Arriagada Gallardo, 2020). La movilidad estudiantil, por ejemplo, ha tenido un serio retroceso. Por ello, "la digitalización y uso de las herramientas tecnológicas" (Fairlie, et al., 2021, p. 8) ha resarcido el déficit de la movilidad al promover escenarios de convivencia, aprendizaje y experiencia de diálogo e intercambio en entornos internacionales. Hoy por hoy, "aumentar la cooperación internacional" (p.9) no sólo es posible gracias a la promoción de esta nueva conciencia y sensibilidad educativas, sino que es un reto que se plantea desde el proceso de digitalización acelerada; es decir, ante una mayor virtualización de la Universidad, la cooperación internacional puede dinamizar la vinculación con unos impactos institucionales, sociales y económicos sin precedentes.

En este contexto de transformación digital surge la vinculación digital como una estrategia que permite replantear la comunicación institucional de la Universidad con la sociedad y dinamizar la interacción virtual en torno a contenidos menos densos o complicados lo que conlleva a convocar una serie más amplia y heterogénea de usuarios. Esta estrategia recae en el uso de las plataformas para la organización de reuniones de trabajo $\mathrm{y}$ eventos multitudinarios desde diferentes escalas y con la posibilidad de que las redes sociales las puedan transmitir en tiempo real. Estas mismas redes sociales -Facebook, Instagram, Twitter- y otras herramientas Youtube, Periscope, Tik Tok - pueden compartir contenidos de forma masiva, recurrente y recursiva. Masiva porque 
no existe un número limitado de usuarios-destino ni tampoco se limita a la comunidad universitaria, sino que involucra usuarios de otras universidades nacionales e internacionales. Es recurrente porque se puede volver a compartir la publicación, editarla o ser retomada por otra instancia universitaria con otros fines institucionales. Es recursiva porque tiene un efecto multiplicador debido a que los usuarios tienden a compartir publicaciones que les interpela lo que provee un alcance sumamente superior porque cada usuario interactúa en/con su propia comunidad virtual que es alimentada cada vez que circula dichos contenidos.

La vinculación digital no debe ser confundida con la comunicación que se despliega en los medios electrónicos -correo electrónico- o digitales -redes sociales- porque tiene un límite que interrumpe la circularidad que es el principio básico en las estrategias digitales de la vinculación. Tampoco lo es porque implica un fenómeno dinámico de retroalimentación que diluye la rigidez institucional y, con ello, se promueve una especie de democracia digital -que había vislumbrado Pierre Lévy hace tres décadas a partir de su concepto de ciberculturaal presuponer la intervención de actores externos -no pertenecientes a la propia Universidad-en la revitalización de la vida sociocultural, y el enriquecimiento de sus procesos cotidianos. La vinculación digital, a diferencia de la comunicación institucional vía remota, asume que lo que se comparte con la comunidad es el principio de un bucle ilimitado, asume una naturaleza enredada que merece ser realimentada, renovada y reincorporada al circuito ininterrumpido de la producción de contenidos digitales. Por ello, la vinculación digital podría ser el rostro visible de un proceso todavía insondable aunque se observa desde esta trialéctica formada por la participación social, la cooperación internacional y la diplomacia intercultural.

\section{| Conclusiones}

En este trabajo se ha realizado una revisión conceptual y teórica de la Responsabilidad Social Universitaria y cómo desde este paradigma institucional se visualiza la vinculación concebida como participación social; es decir, dejando atrás la extensión e involucrando activamente a las comunidades en el proceso de diálogo e interacción de saberes, haberes y haceres, promoviendo una Universidad de carácter social con un espíritu comunitario. Desde la premisa de la participación social se ha hecho hincapié en la importancia de la internacionalización como un segundo estadio de la vinculación, subrayando el papel de la diplomacia cultural e intercultural en el ámbito de la cooperación internacional. Aunado a lo anterior, y como cosecha de la pandemia del covid-19, se ha descubierto las Tecnologías de la Información, Comunicación, Conocimiento y Aprendizaje Digitales (TICCAD) como un insumo de la vinculación digital que dinamiza y fortalece la participación social de la Universidad en los entornos internacionales y globales.

Un texto de esta naturaleza no puede ser concluido de forma positiva, sino planteando una serie de cuestionamientos que responden en forma de preguntas a los retos actuales. ¿Cómo será este retorno a la nueva normalidad, bajo qué secuencias discursivas, institucionales, híper-tecnológicas, socioculturales, la nueva sociedad digital del conocimiento abrirá las puertas de este nuevo universo? ¿Qué rostros de lo humano arrojará el nuevo espacio de la convivencia, la diversidad social y cultural, la inclusión y la igualdad de género en este marco de virtualización acelerada? La Universidad -en tanto que naturaleza expuesta que se cuestiona a sí misma y sus entornos (Derrida, 2010, p. 14) - tendrá que asumir esta infinidad de desafíos tanto al interior como al exterior, reformulando sus funciones, vinculándose con los distintos sectores de la sociedad, interactuando con una comunidad universitaria reticente 
a los cambios institucionales, aunque absorbidos por las transformaciones vitales, psíquicas, anímicas y culturales.

La Universidad del tercer milenio, como se ha planteado en este texto, abre sus aulas al exterior, poniendo su agenda al servicio de la sociedad y sus comunidades, sin renunciar, por ello, a su autonomía. Esta Universidad deberá iniciar esta expedición si es que desea formar parte no sólo de la Nueva Escuela Mexicana, sino enfrentar las terribles adversidades que la Agenda 2030 de la ONU exige cotidianamente. La Responsabilidad Social, la participación social y comunitaria, la diplomacia cultural, la cooperación internacional, la vinculación digital son un sustrato de esta deuda inmaterial que la Universidad tiene con el presente, con lo que está por venir; pero, sobre todo, es una "profesión de fe, un compromiso, una promesa, una responsabilidad asumida" (Derrida, 2010, p.21) consigo misma.

\section{| Notas}

i Acerca de las universidades cristianas, Frederick Copleston (2011, p. II-180) afirma: "Las Universidades, para estar constituidas como tales, necesitaban recibir una Carta formal, bien del papa o bien del emperador [...] o, más tarde, de los reyes. Esas Cartas conferían considerables privilegios a los profesores y alumnos, privilegios que eran celosamente guardados. Los dos privilegios más importantes eran los de jurisdicción interna [...] y el poder de conferir el grado, que llevaba consigo la licencia para enseñar", por lo que aplicar dichos requisitos a la constitución de las universidades en el oriente islámico es un error de perspectiva.

"ii Recordemos que Edgar Morin (1999) había impulsado Los siete saberes necesarios para la educación del futuro (1999) donde exhibía los errores y las ilusiones del conocimiento: los errores mentales, los errores intelectuales, los errores de la razón y las cegueras paradigmáticas. Para ello, nos mostraba los principios de un conocimiento pertinente basado en el contexto, lo global como relación entre el todo y las partes, lo multidimensional y lo complejo que haga posible enseñar la condición humana en medio de la incertidumbre. Sin embargo, Morin advertía de que era necesario, también, enseñar la comprensión más allá del egocentrismo, el etnocentrismo, el sociocentrismo y el espíritu reductor puesto que es vital una ética de la comprensión que dé cuenta de la complejidad humana desde una dimensión planetaria. En otras palabras, transitamos de la civilización global hacia una conciencia de la humanidad como destino planetario. En esta misma coyuntura de Morin, se mueve el sociólogo portugués Boaventura de Sousa Santos (2012) cuando reivindica una epistemología de la visión donde el conocimiento es la base para la erradicación de la sociología de las ausencias, establecer la solidaridad como una forma de conocer y reconocer la alteridad, huir de la inferiorización como un elemento de observar la otredad en su radicalidad extrema en aras de vislumbrar una auténtica sociología de las emergencias; es decir, la irrupción de los sujetos que habían sido invisibilizados, marginados y excluidos del mundo.

iii Luis Alberto Malagón Plata sugiere el concepto de "región de aprendizaje entendida como la delimitación de espacios interactivos de aprendizaje en la vinculación universidad-región".

iv Se refiere a las comunidades con las cuales la Universidad co-construye un proceso complejo de aprendizaje que satisface a ambos. Este espacio de e-co-aprendizaje alude a la ecología de saberes y perspectivas que nos alerta en que los conocimientos universitarios no son los únicos o los privilegiados a los que se llevan a cabo en la comunidad (extensión), en el pueblo o en la ranchería. En la acepción "comunidades de e-co-aprendizaje" yace un deseo de reconstruir un tejido democrático y equitativo donde reine el respeto mutuo a las diferencias, la cooperación social y la responsabilidad intercultural y comunitaria además del impulso a la economía social y solidaria de las localidades y de la región. En consecuencia, "[e]l campus universitario no tendrá límites y el aprendizaje será durante toda la vida" (Malagón Plata, 2006, p.88).

"El vocablo "tik tok" nos hace pensar en el sonido reiterativo de un reloj de arena, un cronómetro que nos indica que estamos al borde del colapso, de la hora fatal, del momento del estallido. Esta herramienta pone el énfasis en la disolución del elemento del tiempo en el consumo de contenidos difundidos a través de esta herramienta y, al mismo tiempo, la incapacidad de recordarlos debido a su poca "esencia" o envergadura temática y a la extensa variedad de su producción. 


\section{| Referencias}

Arriagada Gallardo, F. (2020). El desafío de la vinculación con el medio en tiempos de pandemia Covid-19. Ciencia y Enfermería, 26(11). https://doi.org/h959

Asociación Nacional de Universidades e Instituciones de Educación Superior [ANUIES] (2012). Inclusión con responsabilidad social: Una nueva generación de políticas en educación superior. Editorial Anuies

Augé, M. (2015). Los nuevos miedos (Bixio, A. Trad.). Paidós.

Bauman, Z. (2013). La cultura en el mundo de la modernidad líquida. (Mosconi, L. Trad.). Fondo de Cultura Económica. (Obra original publicada en 2011).

Chebel, M. (2011). El islam. Historia y modernidad (MillánRisco, A. Trad.). Editorial Paidós.

Copleston, F. (2011). Historia de la filosofía. Vol 1: De la Grecia antigua al mundo cristiano. Tomo I Grecia y Roma. Tomo II De San Agustín a Escoto (García-De la Mora, F. Trad.). Editorial Planeta.

Derrida, J. (2010). Universidad sin condición (De Peretti, C \& Vidarte, P. trads.). Editorial Mínima Trotta. (Obra original publicada en 2001).

Fairlie, A., Portocarrero, J. \& Herrera, E. (2021). Desafíos de digitalización para la internacionalización de la educación superior en los países de la Comunidad andina. Documentos de Trabajo, 46 ( ${ }^{\circ}$ época), Fundación Carolina. https://cutt.ly/RTOukJT

Giddens, A. (2002). Consecuencias de la modernidad (LizónRamón Trad.). Editorial Alianza.

Hernández-Leal, E. J. \& Duque-Méndez, N. D. \& MorenoCadavid, J. (2017). Big Data: una exploración de investigaciones, tecnologías y casos de aplicación. TecnoLógicas, 20(39), 15-38. https://doi.org/ghmjwn

Hermoso Villaverde, B. (2021). Entrevista a Pierre Lévy. El País. 26 de junio de 2021. https://cutt.ly/VTOubbj

Huang, F. (2007). L'internationalisation de l'enseignement supérieur à l'ère de la mondialisation : ses répercussions en Chine et au Japon en OCDE. Politiques et gestion de l'enseignement supérieur, 19(1),
49-64. https://cutt.ly/STOunV6

Knight, J. (2003). Interview de Jane Knight. IMHE Info, (1),

\section{https://cutt.ly/dCafu5V}

Laïdi, Z. (1999). Un mundo sin sentido (Ferreiro, J. Trad.). Fondo de Cultura Económica. (Obra original publicada en 1994).

Lévy, P. (2007). Entrevista a Pierre Lévy Sociología Contemporánea. (Hernández-Pérez, C. Trad.) https:// cutt.ly/sTOuTFJ

Malagón Plata, L. A. (2006). La vinculación UniversidadSociedad desde una perspectiva social. Educación y educadores, 9(2), 79-93. https://cutt.ly/gTOuSXo

Mesmoudi, M. (2021). Hacia la internacionalización. Universidad Autónoma de Baja California Sur. https:// cutt.ly/pYX5yU1

Morin, E. (1999). Los siete saberes necesarios para la educación del futuro (Vallejo-Gómez, M. Trad.). UNESCO. https://cutt.ly/9TOuUpu

Nattier, Ch. (2015). La diplomatie publique et culturelle de demain : nouvelles stratégies pour de nouveaux défis, Association Internationale des études québecoises. https://cutt.ly/tTOuPNs

Ortega y Gasset, J. (2002). El tema de nuestro tiempo. La rebelión de las masas (4a ed.) Editorial Porrúa.

Paz, O. (2014). Obras Completas II. Excursiones e incursiones. Dominio Extranjero. Fundación y disidencia. Dominio Hispánico (2a ed.). Fondo de Cultura Económica. (Obra original publicada en 1994).

Rodríguez Barba, F. (2015). Diplomacia cultural. ¿Qué es y qué no es? Espacios Públicos, 18(43), 33-49. https:// cutt.ly/WTOuF6x

Secretaría de Educación Pública [SEP]. (2019). Agenda Digital Educativa, SEP. https://cutt.ly/aTOuHtb

Sousa Santos, B. (2012). Una epistemología del sur: la reinvención del conocimiento y la emancipación social (Gandarilla Salgado, J.G. ed.). Editorial XXI \& Editorial CLACSO.

Vallaeys, F. (2018). Las diez falacias de la Responsabilidad Social Universitaria. Revista Digital en Investigación 
y Docencia Universitaria, 12(1), 34-58. https://doi.org/

$\underline{\mathrm{h} 8 \mathrm{qk}}$

Unión de Responsabilidad Social Universitaria Latinoamericana [URSULA]. (15 de marzo de 2017). Conferencia ¿Por qué la Responsabilidad Social Universitaria no es Extensión Social? [Video]. Youtube. https://cutt.ly/GTOuCHh

Wallerstein, I. (2003). Crítica del sistema-mundo capitalista (Estudio y entrevista de Carlos Antonio Aguirre Rojas). ERA. 